Check for updates

Cite this: RSC Adv., 2019, 9, 38438

Received 9th September 2019

Accepted 11th November 2019

DOI: $10.1039 / c 9 r a 07240 c$

rsc.li/rsc-advances

\section{Cytochalasin Z11 inhibits RANKL-induced osteoclastogenesis via suppressing NFATc1 activation $\uparrow$}

\author{
Lu Wang, $\ddagger^{\mathrm{a}}$ Kai Chen, + $^{\mathrm{b}}$ Jianbo He, ${ }^{\mathrm{bc}}$ Jacob Kenny, ${ }^{\mathrm{b}}$ Yu Yuan, bd Junhao Chen, ${ }^{\mathrm{b}}$ \\ Qian Liu, ${ }^{\text {befg }}$ Renxiang Tan, ${ }^{\mathrm{h}}$ Jinmin Zhao ${ }^{\text {efg }}$ and Jiake Xu (DD *be
}

Excessive osteoclastogenesis and enhanced bone resorption are pathological hallmarks for bone diseases including osteolytic diseases, osteoporosis, and arthritis. Treatments targeting highly activated osteoclasts are regarded as promising therapies for osteoclast-related bone disorders. Cytochalasins are known as secondary metabolites of fungi and exhibit a variety of biological activities in cell biology and medicine. Cytochalasin Z11 (CytoZ11) was previously isolated from the Endothia gyrosa through solid substrate culture and showed therapeutic potential for leukaemia. However, the effects of CytoZ11 on osteoclasts currently remain unclear. Herein, CytoZ11 was found to be able to attenuate RANKL (receptor activator of nuclear factor- $\mathrm{B}$ ligand)-induced osteoclastogenesis and bone resorptive activity dose-dependently. CytoZ11 could also inhibit mRNA expression of osteoclast-specific genes such as Ctr, Acp5, and Ctsk. Furthermore, CytoZ11 was demonstrated to suppress NFATC1 activation, which is due to the attenuation

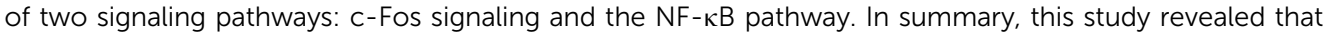
CytoZ11 may become a prospective drug for osteoclast-related disease by inhibiting osteoclast formation and function.

\section{Introduction}

The equilibrium between bone resorption (by osteoclasts) and bone formation (by osteoblasts) is critical to maintain bone homeostasis throughout a lifetime. ${ }^{1}$ Excessive osteoclast activity and/or reinforced bone resorption may cause a wide range of osteolytic diseases such as osteoporosis, arthritis, and periprosthetic osteolysis after joint replacements..$^{2-4}$ In particular, osteoporosis is a common degenerative disease in the aging population, which is associated with fragility fractures,

${ }^{a}$ Department of Orthopaedics, The First Affiliated Hospital of Wenzhou Medical University, Zhejiang Province, China

${ }^{b}$ School of Biomedical Sciences, University of Western Australia, Perth 6009, WA, Australia.E-mail: jiake.xu@uwa.edu.au

${ }^{c}$ Guangzhou University of Chinese Medicine, Guangzhou, Guangdong Province, China

${ }^{d}$ School of Physical Education and Sports Science, South China Normal University, Guangzhou, Guangdong, China

${ }^{e}$ Research Centre for Regenerative Medicine, Guangxi Medical University, Guangxi Province, China

${ }^{f}$ Guangxi Key Laboratory of Regenerative Medicine, Guangxi Medical University, Guangxi, China

IInternational Joint Laboratory on Regeneration of Bone and Soft Tissues, Guangxi Medical University, Guangxi, China

${ }^{h}$ The State Key Laboratory of Pharmaceutical Biotechnology, Institute of Functional Biomolecules, Nanjing University, Nanjing, China

$\dagger$ Electronic supplementary information (ESI) available. See DOI: $10.1039 / \mathrm{c} 9 \mathrm{ra} 07240 \mathrm{c}$

‡ Co-first author: Lu Wang and Kai Chen contributed equally to this work. significant mortality and morbidity, and results in a huge monetary cost to economies. ${ }^{5}$

Osteoclasts, originating from the monocyte/macrophage lineage, are giant cells characterized by multi-nuclei and have the unique ability to "eat" bone by secreting enzymes and acid to degrade the mineralized bone matrix. ${ }^{6}$ Osteoclastogenesis requires the presence of two well-known crucial factors, M-CSF (macrophage-colony-stimulating factor) and RANKL (receptor activator of nuclear factor- $\kappa \mathrm{B}$ ligand). Upon interacting with its receptor-c-fms (colony-stimulating factor 1 receptor), M-CSF supports the survival and proliferation of monocytes/macrophages. ${ }^{7}$ RANKL is expressed and exported by osteocytes, osteoblasts and bone marrow stromal cells, exhibiting an indispensable role in osteoclast formation and function by binding to its receptor-RANK. ${ }^{8}$

The interaction of RANKL with RANK, which is a transmembrane receptor on the surface of osteoclast precursor cells, initiates osteoclast differentiation. Upon RANKL stimulation, TNF receptor associated factor 6 (TRAF6) and RANK are trimerized to trigger numerous downstream signaling events such as the NF- $\mathrm{B}$ pathway, c-Fos signaling, and subsequent initiation of nuclear factor of activated T cells 1 (NFATc1). ${ }^{9,10}$ RANKLmediated NFATc1 signaling acts as a master switcher for terminal differentiation of osteoclasts, with its activation upregulating a series of osteoclast-specific genes such as Acp5 (acid phosphatase 5), Ctsk (cathepsin K), Ctr (calcitonin receptor) and V-ATPase-d2. ${ }^{\mathbf{1 1}-13}$ Therefore, inhibition of RANKL- 
induced NFATc1 signaling could effectively prevent osteoclast formation and function, which provides a rationale for the management of osteoclast-related bone diseases.

Cytochalasins, derived from secondary fungal metabolites, have a variety of biological functions and are considered to have huge prospect in life science. ${ }^{\mathbf{1 4 , 1 5}}$ Cytochalasin B (CytoB) has an unique antineoplastic activity and increases the life expectancy of leukemic mice. ${ }^{16}$ Cytochalasin D (CytoD) was revealed to induce adipose tissue-derived mesenchymal stem cells (MSCs) to undergo osteogenic differentiation. ${ }^{17}$ However, the biological effects of cytochalasins on osteoclasts still remain unknown. One type of cytochalasins-cytochalasin Z11 (CytoZ11), isolated from Endothia gyrosa through the solid substrate culture, exhibits a therapeutic potential on a leukaemia K562 cell line. ${ }^{\mathbf{1 8 , 1 9}}$ Herein, we identified that Cytoz11 is capable of inhibiting osteoclast formation and resorptive function in vitro. Mechanistically, CytoZ11 affects NFATc1 activation via suppressing both NF- $\mathrm{KB}$ and c-Fos signaling. Taken together, the findings of this study demonstrated that CytoZ11 may represent an innovative candidate for the treatment of bone disorders like osteoporosis by targeting excessive osteoclasts formation and resorption.

\section{Materials and methods}

\subsection{Materials}

CytoZ11 was kindly provided by Prof. Renxiang Tan's lab (Nanjing University, China). ${ }^{18}$ Dimethyl sulfoxide (DMSO) was used to dissolve CytoZ11 to a concentration of $100 \mathrm{mM}$ for main stock. For further dilution to working concentrations, culture medium was used in this study. For the control group, DMSO in the same dilution was prepared. Fetal bovine serum (FBS) and Alpha modified Minimal Essential Medium (a-MEM) were obtained from Thermo Fisher Scientific (Scoresby, Australia). Glutathione $S$-transferase (GST)-rRANKL protein was made in our lab as previously described. ${ }^{20}$ We purchased recombinant M-CSF from R\&D Systems (Minneapolis, USA). Luciferase analysis kit and MTS assay kit were obtained from Promega (Sydney, Australia). Primary antibodies for NFATc1, integrin $\alpha \mathrm{V}$, cathepsin $\mathrm{K}$, and $\beta$ actin were obtained from Santa Cruz Biotechnology (Dallas, CA, USA). We purchased primary antibody for c-Fos from Cell Signalling Technology (Danvers, MA, USA).

\subsection{In vitro osteoclastogenesis assay}

The C57BL/6 mice were euthanized, and fresh bone marrow were obtained by flushing the lower limbs long bones using medium. The methods of tissue culture used in this study is approved by the Animal Ethics Committee (RA/3/100/1244) in University of Western Australia. In order to further purify the bone marrow macrophages (BMMs), cells were incubated in complete medium $(\alpha-\mathrm{MEM}+\mathrm{M}-\mathrm{CSF}+10 \% \mathrm{FBS}+1 \%$ penicillin/ streptomycin) overnight, after which the floating cells were discarded, and non-adherent cells were incubated in complete aMEM for further 3 days. To conduct osteoclastogenesis assay, we plated BMMs $\left(6 \times 10^{3}\right.$ cells per well $)$ into 96 -well plates in triplicate. Cells were all incubated in a humidified incubator $\left(5 \% \mathrm{CO}_{2}, 37{ }^{\circ} \mathrm{C}\right)$ to allow the cells adherence. Next, BMMs in complete medium were treated with both RANKL (50 $\mathrm{ng} \mathrm{ml}{ }^{-1}$ ) and M-CSF (50 ng ml ${ }^{-1}$ ), and various concentrations of CytoZ11 $(0,1,2.5,5,7.5 \mu \mathrm{M})$ were added in the medium. The culture medium was replaced every other day till the osteoclasts formed. Following osteoclastogenesis, the cells were then proceeded to fixation using $2.5 \%$ glutaraldehyde for 10 minutes at room temperature (RT). From the TRAcP staining, osteoclasts and cells with more than 3 nuclei were counted as mature osteoclasts. Immature osteoclast with 3 or less nuclei were also quantified.

\subsection{MTS cell proliferation assay}

To evaluate the cytotoxic effects of Cytoz11 on BMMs, a commercial MTS assay kit was employed. In brief, BMMs $(6 \times$ $10^{3}$ cells per well) were seeded in a 96-well plate and allowed adhering overnight, followed by the treatment of CytoZ11 at various concentrations $(0,1,2.5,5,7.5 \mu \mathrm{M})$ for another 2 days. Next, cells were incubated with $20 \mu \mathrm{l}$ of MTS solution for 2 hours in accordance with the manufacturer's protocol. The absorbance was measured at a wavelength of $490 \mathrm{~nm}$ using a microplate reader (BMG LABTECH, Germany).

\subsection{Hydroxyapatite resorption assay}

A hydroxyapatite resorption assay was performed to evaluate the function of osteoclasts as previously described. ${ }^{21} \mathrm{BMMs}\left(1 \times 10^{5}\right.$ cells per well) were firstly planted on to six-well culture plates (Corning, Inc., Corning, NY) and induced into osteoclast by stimulating with RANKL and MCSF as above. The cells were then collected using cell dislocation solution (Sigma-Aldrich, Sydney, Australia) when osteoclasts just began to form and subsequently seeded onto hydroxyapatite-coated plates (Corning, Inc., NY, USA). Complete medium containing M-CSF and RANKL with CytoZ11 $(0,2.5,7.5 \mu \mathrm{M})$ was added to the plates until mature osteoclasts start to form. In each group, half of wells were rinsed using $10 \%$ bleach solution and then the resorptive area was visualized using a microscope (Nikon corporation). To count the number of osteoclasts in the hydroxyapatite plate, cells in the other half wells were performed for TRAcP staining as described above. The osteoclastic resorption activity was determined by resorption area per osteoclast.

\subsection{NFATc1 and NF- $\quad$ B luciferase assay}

NFATc1 and NF- $\kappa$ B transcriptional activation were evaluated using RAW264.7 cells which were stably transfected with an NFATc1 or NF- $\kappa$ B luciferase reporter construct or an as described previously. ${ }^{22}$ Transfected cells $\left(5 \times 10^{4}\right.$ cells per well for NFATc1, or $1.5 \times 10^{5}$ cells per well for NF- $\kappa$ B) were plated onto 48-well plates. The next day, cells were treated with CytoZ11 at $0,2.5,5,7.5 \mu \mathrm{M}$ for 1 hour prior to the stimulation with RANKL. RANKL was added in the cell culture for 24 hours or 8 hours in NFATc1 or NF- $\kappa$ B luciferase assay respectively. Following the cells lysis, the Promega luciferase kit was used to detect the luminance intensity using a Polar Star Optima luminescence reader (BMG LABTECH, Ortenberg, Germany). 


\subsection{Quantitative polymerase chain reaction (qPCR) analysis}

To assess osteoclast-specific gene expression, qPCR analysis was performed. BMMs $\left(1 \times 10^{5}\right.$ cells per well $)$ were induced into osteoclasts in 6-well plates and treated with or without CytoZ11 $(0,2.5,7.5 \mu \mathrm{M})$. After osteoclasts formed, the extraction of RNA was performed using Trizol reagent as the manufacturer's protocol instructed. The cDNA was transcribed from RNA template $(1 \mu \mathrm{g})$ using an oligo-dT primer and reverse transcriptase (Promega). We use SYBR Green PCR MasterMix to conduct qPCR in the ViiATM 7 Real-Time PCR System (Thermo Fisher Scientific, USA). The primers used in this study are showed in Table 1.

The program parameters were set: following $94{ }^{\circ} \mathrm{C}$ for 10 minutes, 40 cycles of $95{ }^{\circ} \mathrm{C}$ for 15 seconds, $60{ }^{\circ} \mathrm{C}$ for 60 seconds, and $72{ }^{\circ} \mathrm{C}$ for 40 seconds, an elongation step of 5 minutes at $72{ }^{\circ} \mathrm{C}$ was performed. All experiments were run in triplicate. The housekeeping gene $A c t b$ worked as a control gene.

\subsection{Western Blot assay}

Western blot assay was used to investigate the signaling pathways affected by CyzoZ11. BMMs were seeded onto a 6-well plate at a density of $1 \times 10^{5}$ per well. The cells were stimulated using RANKL with CytoZ11 at the concentrations of 0 and $7.5 \mu \mathrm{M}$ for the indicated times $(0,1,3$, or 5 days) and then lysed using RIPA lysis and extraction buffer for protein collection. The protein was separated by running SDS-PAGE and then transferred onto a nitrocellulose (NC) membrane. To avoid unspecific binding, the membranes were incubated using 5\% skim milk for 1 hour at room temperature. The proteins of interest were incubated with primary antibodies overnight with gentle rocking. Next, the membranes were rinsed three times using TBST and probed with appropriate secondary antibodies conjugated with horseradish peroxidase (HRP) for 2 hours. Enhanced chemiluminescence (ECL) kit (PerkinElmer, MA) was used to detect the protein bands of interest. Image-quant LAS 4000 (GE Healthcare) was used to detect specific antibodies reactivity.

\subsection{Data analysis}

The results in this study were shown as the mean \pm standard deviation (SD). All experiments were performed 3 or more times.

Table 1 Primer sequence for $\mathrm{qPCR}^{a}$

\begin{tabular}{ll}
\hline Gene & Primer sequence $\left(5^{\prime}-3^{\prime}\right)$ \\
\hline \multirow{2}{*}{ Actb } & F: $5^{\prime}$-CACCCGCGAGCACAGCTTCTT-3' \\
& R: $5^{\prime}$-CCACCATCACACCCTGGTGCCT-3' \\
Acp5 & F: $5^{\prime}$-TGTGGCCATCTTTATGCT-3' \\
& R: $5^{\prime}$-GTCATTTCTTTGGGGCTT-3' \\
Ctsk & F: $5^{\prime}$-GGGAGAAAAACCTGAAGC-3' \\
& R: $5^{\prime}$-ATTCTGGGGACTCAGAGC-3' \\
Ctr & F: $5^{\prime}$-TGGTTGAGGTTGTGCCA-3' \\
& R: $5^{\prime}$-CTCGTGGGTTTGCCTCATC-3'
\end{tabular}

${ }^{a}$ Abbreviations: Actb, actin beta; Acp5, acid phosphatase 5, tartrate resistant; Ctsk, cathepsin K; Ctr, calcitonin receptor; F, forward; R, reverse.
Data analyses were made using Student's $t$ test. In the results, $p$ value $<0.05$ was considered to have significant difference.

\section{Results}

\subsection{CytoZ11 suppresses osteoclastogenesis in vitro}

To determine whether CytoZ11 has effects on the osteoclast formation, CytoZ11 $(0,1,2.5,5,7.5 \mu \mathrm{M})$ were used to treat BMMs when they are induced with RANKL and M-CSF. It was found that CytoZ11 is capable of inhibiting osteoclastogenesis dose-dependently (Fig. 1A). The number of TRAcP-positive osteoclasts was reduced significantly in the presence of CytoZ11 of the concentrations of $5 \mu \mathrm{M}$ and $7.5 \mu \mathrm{M}$ (Fig. 1B). CytoZ11 was reported to have cytotoxicity on cancer cells. ${ }^{18}$ To assess whether CytoZ11 is cytotoxic to BMMs, MTS assays were conducted to further evaluate cell proliferation after $48 \mathrm{~h}$ treatment. The results did not show any cytotoxicity on BMMs at any concentrations of CytoZ11 as indicated in Fig. 1C.

\subsection{CytoZ11 suppresses RANKL-induced osteoclast resorptive function and genes expression}

Hydroxyapatite-coated plates were used to determine whether CytoZ11 can suppress mature osteoclastic bone resorption. As shown in Fig. 2A-C, osteoclast number and hydroxyapatite resorption area were both significantly reduced due to the treatment of CytoZ11, particularly at the concentration of 7.5 $\mu \mathrm{M}$. We evaluated its effects on the expression of osteoclastspecific genes (Ctsk, Acp5, and Ctr). The results showed that RANKL dramatically up-regulated the expression of these genes, while the addition of CytoZ11 $(7.5 \mu \mathrm{M})$ was able to attenuate this up-regulation (Fig. 3A-C). Therefore, CytoZ11 was thought to suppress osteoclast bone formation and function via inhibiting the osteoclast-specific genes expression levels.

\subsection{CytoZ11 suppresses RANKL-mediated NFATc1 signaling}

NFATc1 is considered as a main "switcher" for osteoclastogenesis. ${ }^{12}$ To test the effect of CytoZ11 on NFATc1 transcriptional activation, NFATc1 luciferase reporter assay was performed using transfected RAW264.7 as described above. The results showed that RANKL dramatically enhanced the transcriptional activity of NFATc1, which was inhibited by CytoZ11 dose-dependently (Fig. 4B). These results were consistent with the results in western blot assay. After BMMs were stimulated with RANKL on day 3, the protein level of NFATc1 was substantially upregulated but was abrogated with the treatment of CytoZ11 (7.5 $\mu \mathrm{M})$ (Fig. 5A and C). CTSK and integrin $\alpha \mathrm{V}$ act as two important downstream proteins of NFATc1 activation and are required for osteoclast formation and function. ${ }^{\mathbf{1 0 2 3}}$ The protein expression level of CTSK and integrin $\alpha \mathrm{V}$ were examined using western blot assay. The decreasing trend of the protein expression was observed after CytoZ11 treatment (Fig. 5A). A significant reduction of CTSK was observed on day 5 (Fig. 5D) whereas a significant reduction of integrin $\alpha \mathrm{V}$ was observed on day 3 (Fig. 5E). Collectively, these results demonstrated that RANKL-induced NFATc1 activation was significantly affected by the treatment of CytoZ11 during osteoclastogenesis. 
A

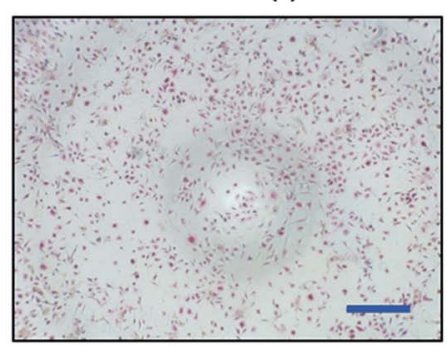

RANKL(-)

RANKL+ CytoZ11 $(2.5 \mu \mathrm{M})$

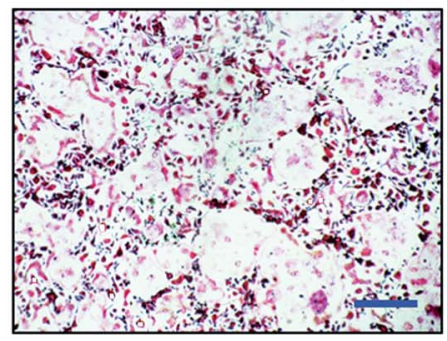

RANKL(+)

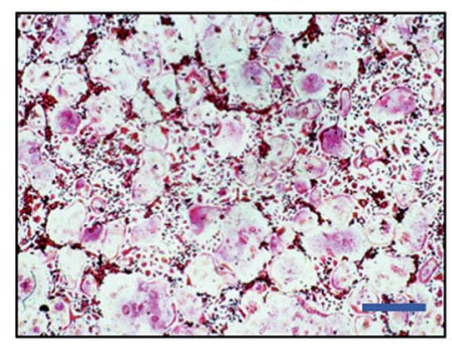

RANKL+ CytoZ11 (5 $\mu \mathrm{M})$
RANKL+ CytoZ11 $(1 \mu \mathrm{M})$

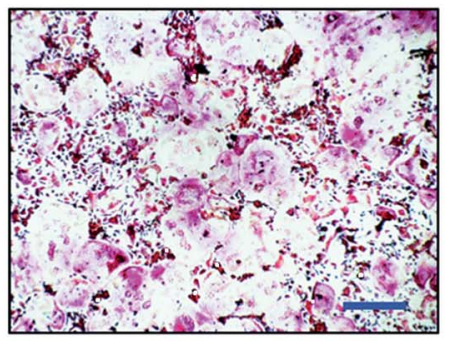

RANKL+ CytoZ11 $(7.5 \mu \mathrm{M})$

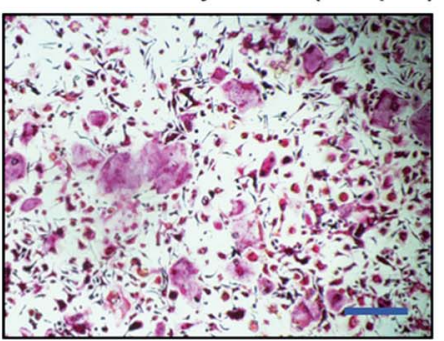

B

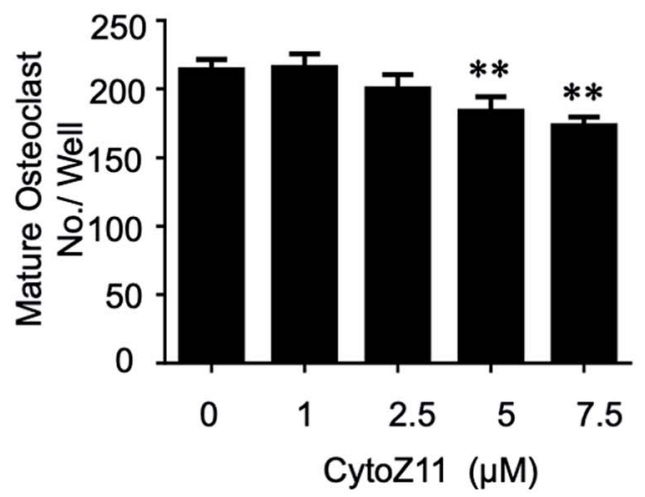

C

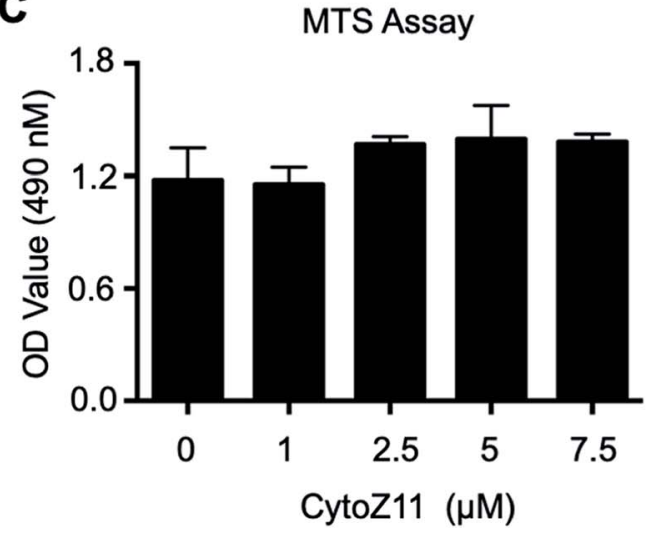

Fig. 1 Cytochalasin Z11 (CytoZ11) inhibited RANKL-induced osteoclastogenesis in vitro dose-dependently. (A) Typical images of osteoclastogenesis in the presence of the different doses of CytoZ11 for 5 days. Cells were then proceeded to fixation and subsequent TRAcP staining. (B) Quantification of TRAcP-positive, multinucleated cells (nuclei $>3$ ) ( $n=3$ per group). (C) The viability of BMMs following 48 hour treatment of CytoZ11 was measured using MTS assay. ${ }^{* *} p<0.01$ relative to RANKL-induced control group. Scale bar $=200 \mu \mathrm{m}$. MTS: 3-(4,5-dimethylthiazol2-yl)-5-(3-carboxymethoxyphenyl)-2-(4-sulfophenyl)-2H-tetrazolium, inner salt; TRACP: tartrate resistant acid phosphatase; RANKL: receptor activator of nuclear factor- $\mathrm{B}$ ligand.

3.4. CytoZ11 inhibits RANKL-induced NF- $\kappa B$ pathway as well as c-Fos signaling

To elucidate the mechanisms underlying the Cytoz11's inhibition on NFATc1 activation, NF- $\kappa \mathrm{B}$ and $\mathrm{c}-\mathrm{Fos}$, two upstream signaling of NFATc1, were further investigated. NF- $\kappa \mathrm{B}$ binds to the NFATc1 promoter and initiates its activation. ${ }^{24}$ In consistency with the NFATc1 luciferase reporter assay, initiation of $\mathrm{NF}-\kappa \mathrm{B}$ was detected with the treatment of RANKL at transcriptional level but was attenuated by CytoZ11 of the concentrations at 5 and $7.5 \mu \mathrm{M}$ (Fig. 4A). C-Fos is an upstream factor cooperating with NFATc1 to trigger a transcriptional regulatory cascade. ${ }^{25}$ RANKL-induced c-Fos signaling during osteoclastogenesis was assessed using a western blot assay. The expression of c-Fos was dramatically suppressed with CytoZ11 treatment on day 3 (Fig. 5A and B). In summary, this study demonstrates that CytoZ11 suppressed RANKL-induced NFATc1 activation via attenuating NF- $\kappa \mathrm{B}$ pathway as well as c-Fos signaling (Fig. 6).

\section{Discussion}

Bone remodeling remains a dynamic process and is firmly regulated by osteoclasts and osteoblasts throughout life. ${ }^{\mathbf{1 0 , 2 6}}$ Imbalance between osteoclasts and osteoblasts can affect bone homeostasis thus leading to a variety of bone diseases. Osteoporosis, a common metabolic bone disorder, relates to excessive bone resorption which is caused by the enhanced activity and/or increased formation of osteoclasts. ${ }^{27}$ Targeting osteoclasts may be conducive to providing a treatment for osteoporosis or 
A

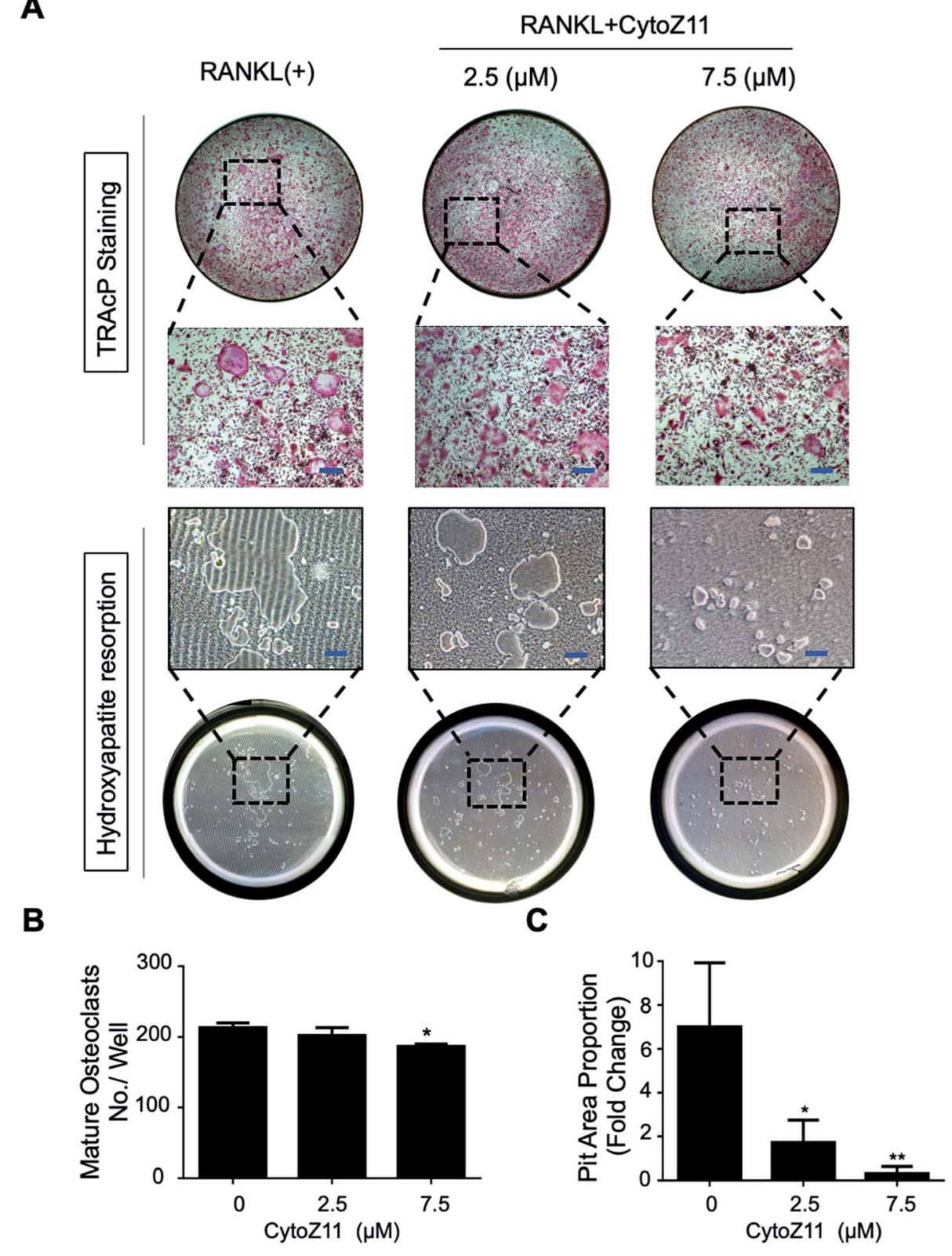

Fig. 2 CytoZ11 suppressed osteoclast resorptive function. (A) Typical images of TRAcP-positive osteoclasts (upper panel) and hydroxyapatite resorption (lower panel) after the CytoZ11 treatment at different concentrations $(0,2.5,7.5 \mu \mathrm{M})$. RANKL-induced osteoclasts were planted into hydroxyapatite-coated plates. (B) Quantification of TRAcP-positive multinucleated cells per well $(n=3)$. (C) Quantification of resorbed hydroxyapatite surface area $(n=3) .{ }^{*} p<0.05, * * p<0.01$ relative to RANKL-induced control group. Scale bar $=200 \mu \mathrm{m}$. CytoZ11: cytochalasin Z11; TRACP: tartrate-resistant acid phosphatase; RANKL: receptor activator of nuclear factor-кB ligand.

osteoclast-related diseases. Over the past two decades, treatments for osteoporosis have made great progress but still remain limited in some respects. Although bisphosphonate (BPs) drugs show efficient clinical outcomes by blocking osteoclast resorption, it increases the incidence of osteonecrosis of the jaw and atypical fractures due to prolonged use. ${ }^{28}$ Parathyroid hormone, a pricy therapeutic peptide which cannot be used orally, is not recommended for long-term treatment because of a potential risk of osteosarcoma. ${ }^{29}$ Therefore, it is essential to develop better alternative treatments which are more cost-effective and safer. Herein, for the first time, CytoZ11 has been revealed to inhibit RANKL-induced osteoclast formation as well as bone resorption, suggesting its potential applications in osteoclast-related bone disorders.

Given the wide range of biological activities of CytoZ11, we first examined whether it has any effect on RANKL-mediated osteoclastogenesis in vitro. CytoZ11 could efficiently inhibit mature osteoclast formation dose-dependently but without affecting the viability or the proliferation of BMMs. Cytoz11 starts to take effect at $2.5 \mu \mathrm{M}$ by showing a decreased trend of osteoclast formation, but reaches a significant point when the concentration increases to $5 \mu \mathrm{M}$ and higher. CytoZ11 at $5 \mu \mathrm{M}$ 

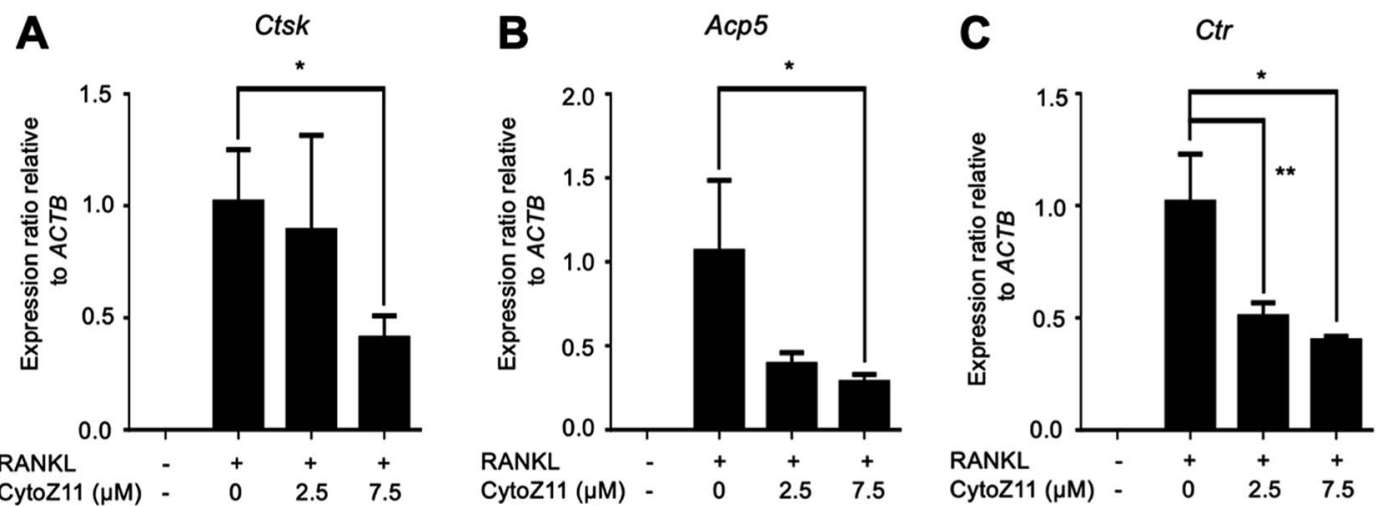

Fig. 3 CytoZ11 suppressed osteoclast-specific genes expression. The qPCR analysis was performed to determine the osteoclast-specific genes, including (A) Ctsk, (B) Acp5, (C) Ctr. The results were normalized to the expression of the housekeeping gene Actb. ${ }^{*} p<0.05,{ }^{* *} p<0.01$ relative to RANKL-induced controls. CytoZ11: cytochalasin Z11; qPCR: quantitative polymerase chain reaction; Ctsk: encoding cathepsin K; Acp5: encoding tartrate-resistant acid phosphatase (TRACP); Ctr: encoding calcitonin receptor; RANKL: receptor activator of nuclear factor- $\kappa B$ ligand.

and $7.5 \mu \mathrm{M}$ is able to significantly inhibit mature osteoclast formation. Interestingly, more immature trap-positive cells were observed following the treatment of cytoZ11 of these two concentrations, indicating the cytoZ11 may also prevent the maturation of the osteoclasts (Fig. S1†). We also found that CytoZ11 was efficient in suppressing bone resorptive activity. With regards to the mechanisms behind these inhibitions, CytoZ11 was found to attenuate NFATc1 activation and the mRNA expression level of genes including Acp5, Ctsk, Ctr. Similarly to the osteoclastogenesis assay, CytoZ11 at the low concentration $(2.5 \mu \mathrm{M})$ is able to affect these osteoclast-specific markers to different extents, but the effects become solid at higher concentration $(7.5 \mu \mathrm{M})$. These results collectively indicated that CytoZ11 could present as a novel beneficial candidate natural compound for bone osteolytic disorders which are due to highly activated osteoclasts.

Following RANKL binding to its receptor RANK, BMMs are mediated to differentiate into osteoclasts as well as to maintain bone resorption of mature osteoclasts. ${ }^{30}$ Recombinant RANK-Fc was shown to be of high affinity to RANKL in vitro and blunted osteoclast formation, this was further evidenced by the results that polyclonal antibody against the RANK extracellular domain can stimulate osteoclast differentiation. ${ }^{31}$ NFATc1, a downstream target of RANKL, plays an important role in the osteoclast terminal differentiation. ${ }^{12} \mathrm{~A}$ previous study has revealed that embryonic stem cells deficient of NFATc1 exhibited a failure to form osteoclasts in the presence of RANKL; in contrast, ectopic expression of NFATc1 induced osteoclast differentiation even without RANKL stimulation. ${ }^{12}$ As a dominator for osteoclast differentiation, NFATc1 also initiates a variety of osteoclast-related genes including Integrin $\alpha \mathrm{V},{ }^{23} \mathrm{Acp} 5,{ }^{12} \mathrm{Ctr},{ }^{10} \mathrm{Ctsk} .{ }^{32}$ Therefore, we investigated the transcriptional activity and protein level of NFATc1 during RANKL-induced osteoclastogenesis using western blot analysis and luciferase gene assay. Our results demonstrated that CytoZ11 could dramatically inhibit NFATc1 activation which is accompanied by the reduced mRNA expression of various osteoclast specific genes such as Acp5, Ctr, and Ctsk.
We next identified the molecular mechanisms of NFATc1 activation. NF- $\mathrm{KB}$ activation was crucial for the induction of NFATc1 autoamplification through interacting with its promotor at the
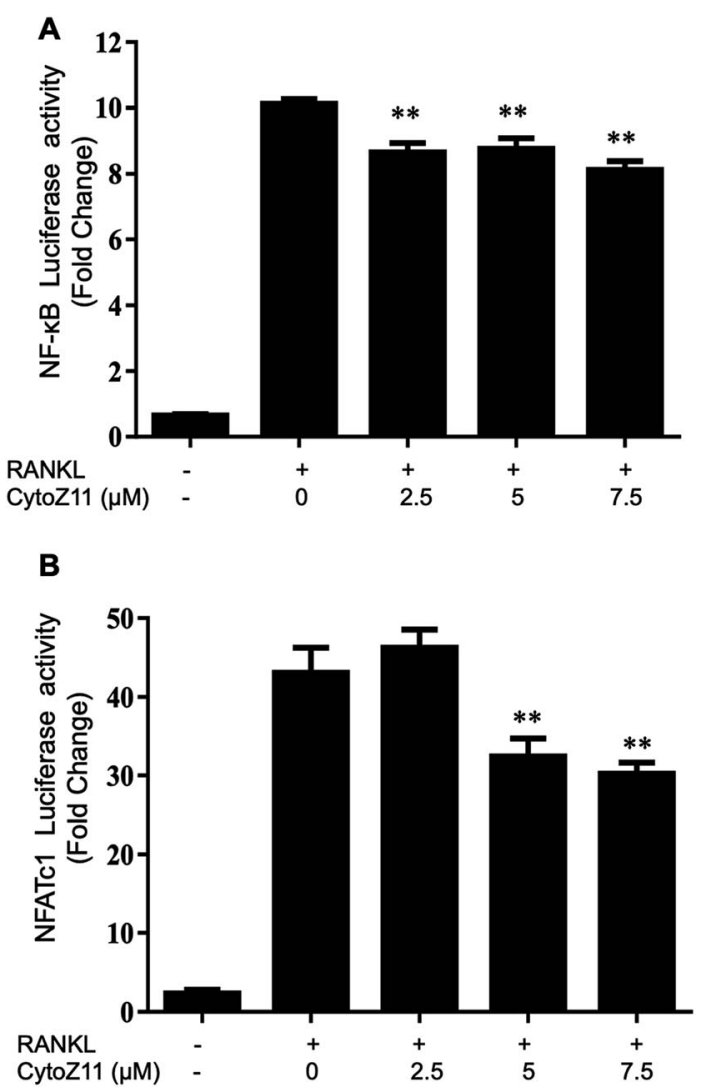

Fig. 4 CytoZ11 affected NF- $\kappa B$ pathway and NFATc1 activation. RAW264.7 cells transfected with a NFATc1 or NF- $\mathrm{BB}$ luciferase construct were pretreated with different doses of CytoZ11 $(0,2.5,7.5$ $\mu \mathrm{M})$ for 1 hour, followed by stimulation with RANKL $\left(50 \mathrm{ng} \mathrm{ml}^{-1}\right)$ for 24 hours or 8 hours; respectively. Luciferase reporter assay was performed to evaluate the transcriptional activity of (A) NF- $\mathrm{B} B$ and (B) NFATc1 $(n=3)$. ${ }^{* *} p<0.01$ relative to RANKL-induced control group. CytoZ11: cytochalasin Z11; NFATc1: nuclear factor of activated T cells 1; NF- $\kappa B$ : nuclear factor $-\kappa B$; RANKL: receptor activator of nuclear factor-kB ligand. 

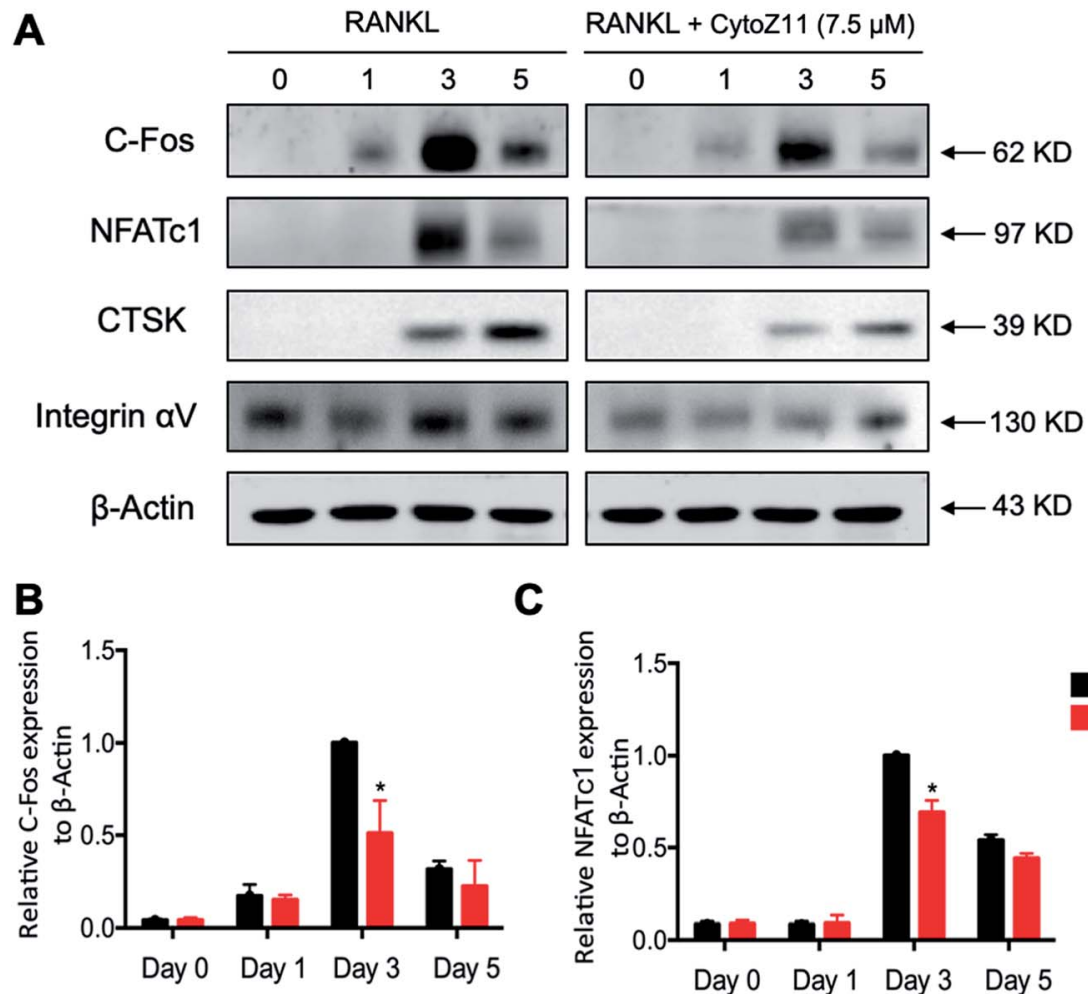

\section{C}

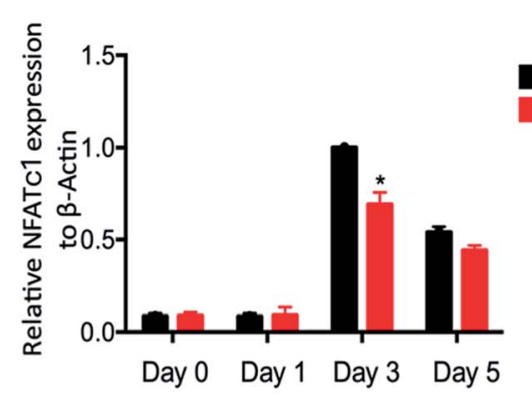

RANKL

RANKL+CytoZ11 $(7.5 \mu \mathrm{M})$

D E
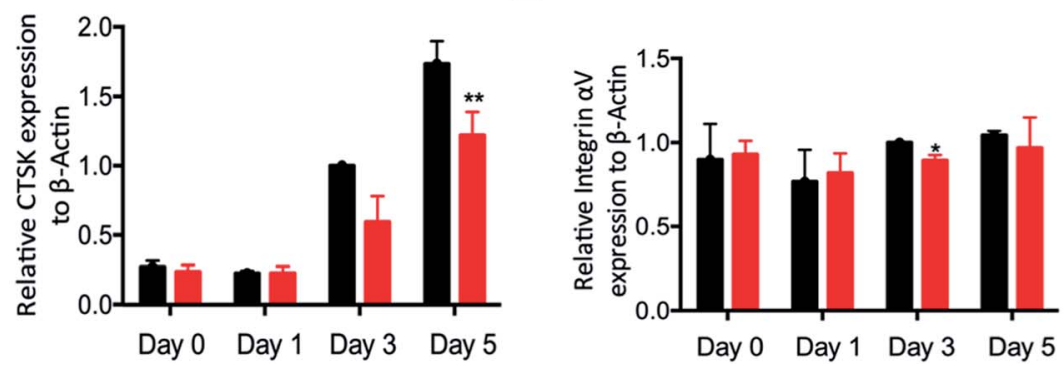

Fig. 5 CytoZ11 attenuated NFATc1 activation, C-Fos signaling. (A) Typical images of the western blot demonstrating the effects of CytoZ11 on the protein expression of NFATc1, c-Fos, Ctsk, integrin $\alpha \mathrm{V}$ during RANKL-induced osteoclastogenesis. Quantification of ratios of band intensity of (B) C-Fos, (C) NFATc1, (D) CTSK, and (E) integrin $\alpha$ V relative to $\beta$-actin. $* p<0.05, * * p<0.01$ relative to RANKL-induced control group. CytoZ11: cytochalasin Z11; RANKL: receptor activator of nuclear factor-кB ligand; NFATc1: nuclear factor of activated T cells 1.

early phase of RANKL-induced osteoclastogenesis. ${ }^{11,33}$ Loss of NF$\kappa \mathrm{B}$ signaling incurred compromised osteoclastogenesis and resulted in an osteopetrotic bone phenotype in mice. ${ }^{34}$ The results of the NF- $\kappa$ B luciferase gene assay indicated that the NF- $\kappa \mathrm{B}$ transcriptional level was inhibited by the treatment of CytoZ11, which may account for the subsequent suppression of NFATc1 activity. However, in this study, we found that CytoZ11 at the concentration of $2.5 \mu \mathrm{M}$ significantly attenuated the NF- $\mathrm{kB}$ signaling but failed to affect the NFATc1 activation. As NFATc1 acts as a "master switcher" for osteoclastogenesis, this is also consistent with the result that osteoclast formation was not inhibited by $2.5 \mu \mathrm{M}$ CytoZ11 in osteoclastogenesis assay. Given the auto-amplification of NFATc1 also largely result from the upstream signaling including $\mathrm{Ca}^{2+}$ signaling ${ }^{35}$ and c-Fos signaling, ${ }^{11}$ whether CytoZ11 of the low concentration is able to affect these two signaling remain to be further investigated. Thus, the suppression of Cytoz11 on NFATc1 activation is at least partly due to the suppression of the NF- $\mathrm{B}$ activation.

C-Fos, a crucial constituent of dimeric factors AP-1, is known to act as a direct transcriptional regulator for the activation of NFATc1. ${ }^{11,36}$ Precursor cells lacking the c-Fos gene were incapable of activating NFATc1 by RANKL and failed to transform into the osteoclast lineage. ${ }^{\mathbf{1 2}}$ Furthermore, an active form of NFATc1 transformed into c-Fos deficient cells rescued osteoclastogenesis and bone resorption. ${ }^{25}$ In accordance with the suppressive effect of CytoZ11 on NFATc1 activation, CytoZ11 was found to dramatically reduce the protein level of c-Fos during RANKL-induced osteoclastogenesis. We thus reasoned CytoZ11's inhibition on NFATc1 was partly through its effect on c-Fos signaling. 


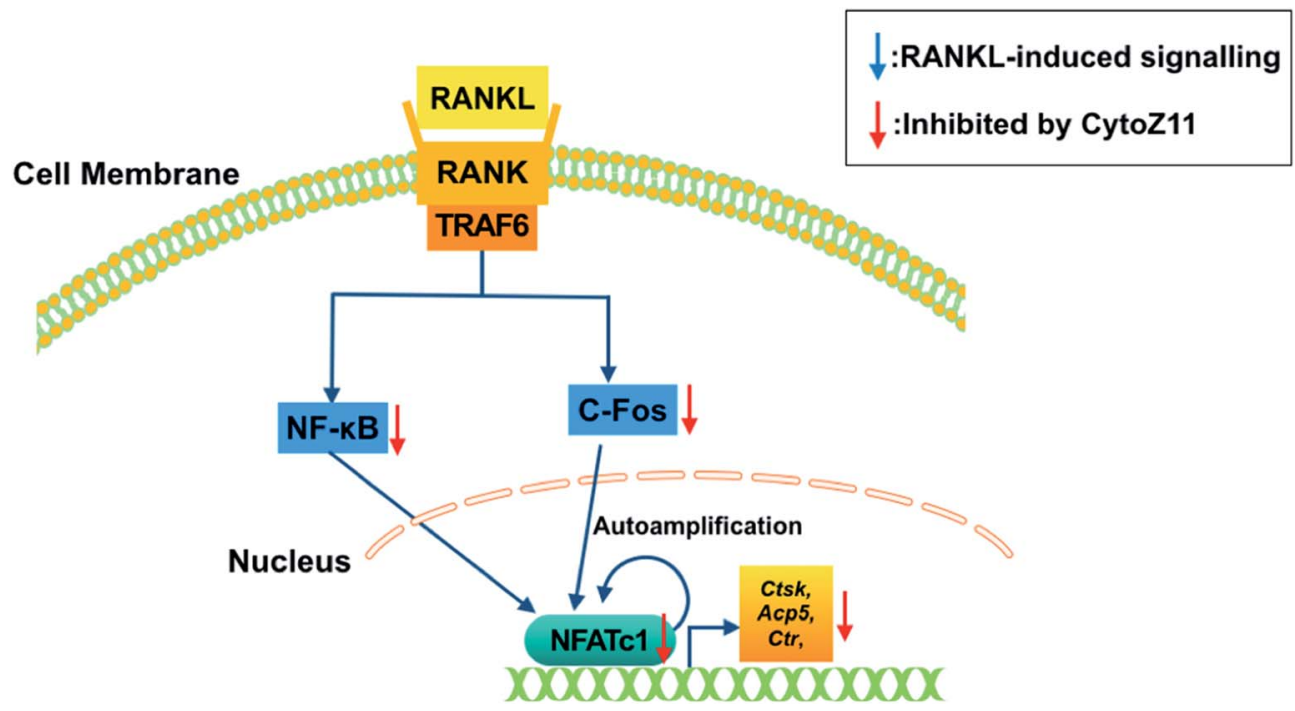

Fig. 6 A proposed scheme of the impacts of CytoZ11 on RANKL-induced osteoclastogenesis. CytoZ11 inhibited $c-F o s$ and NF- $\kappa B$ signaling pathways, resulting in the suppression of NFATc1 activation, as well as the down-regulation of osteoclast-specific genes such as Ctsk, Acp5, and Ctr. Acp5: encoding tartrate-resistant acid phosphatase (TRACP); CytoZ11: cytochalasin Z11; Ctsk: encoding cathepsin K; Ctr: encoding calcitonin

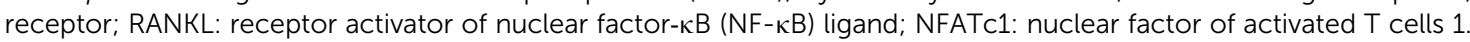

\section{Conclusion}

In conclusion, this study revealed that CytoZ11 is able to inhibit RANKL-induced osteoclast formation and bone resorption by affecting NFATc1 activation. This is achieved by attenuating two signaling pathways, including c-Fos signaling and NF- $\kappa \mathrm{B}$ pathways. Therefore, it is suggested that CytoZ11 may serve as a promising natural compound for the development of a therapeutic approach to counter osteoclast-related bone diseases. However, the therapeutic effects in vivo are still needed to be further determined by using preclinical animal models.

\section{Conflicts of interest}

All authors declare that they have no conflicts of interest.

\section{Acknowledgements}

This study was supported by grants from the Australian National Health and Medical Research Council (NHMRC, No. 1107828, 1127156, 1163933), and Wenzhou Municipal Sci-Tech Bureau Program (Y20190276). This study is also supported by the UWA-CHINA Collaborative Award (2017). Kai Chen is supported by Australian Government Research Training Program Scholarship. We acknowledge the facilities and technical assistance of the Centre for Microscopy, Characterization \& Analysis, The University of Western Australia.

\section{Reference}

1 J. Kular, J. Tickner, S. M. Chim and J. Xu, An overview of the regulation of bone remodelling at the cellular level, Clin. Biochem., 2012, 45, 863-873.
2 S. L. Teitelbaum, Osteoclasts: what do they do and how do they do it?, Am. J. Pathol., 2007, 170, 427-435.

3 Y. Abu-Amer, I. Darwech and J. C. Clohisy, Aseptic loosening of total joint replacements: mechanisms underlying osteolysis and potential therapies, Arthritis Res. Ther., 2007, 9(suppl. 1), S6.

$4 \mathrm{M}$. Zaidi, Skeletal remodeling in health and disease, Nat. Med., 2007, 13, 791-801.

5 N. Harvey, E. Dennison and C. Cooper, Osteoporosis: impact on health and economics, Nat. Rev. Rheumatol., 2010, 6, 99105.

6 G. D. Roodman, Cell biology of the osteoclast, Exp. Hematol., 1999, 27, 1229-1241.

7 F. Arai, T. Miyamoto, O. Ohneda, T. Inada, T. Sudo, K. Brasel, et al., Commitment and differentiation of osteoclast precursor cells by the sequential expression of c-Fms and receptor activator of nuclear factor kappaB (RANK) receptors, J. Exp. Med., 1999, 190, 1741-1754.

8 T. Nakashima and H. Takayanagi, New regulation mechanisms of osteoclast differentiation, Ann. N. Y. Acad. Sci., 2011, 1240, E13-E18.

9 T. Suda, N. Takahashi, N. Udagawa, E. Jimi, M. T. Gillespie and T. J. Martin, Modulation of osteoclast differentiation and function by the new members of the tumor necrosis factor receptor and ligand families, Endocr. Rev., 1999, 20, 345-357.

10 W. J. Boyle, W. S. Simonet and D. L. Lacey, Osteoclast differentiation and activation, Nature, 2003, 423, 337-342.

11 M. Asagiri, K. Sato, T. Usami, S. Ochi, H. Nishina, H. Yoshida, et al., Autoamplification of NFATc1 expression determines its essential role in bone homeostasis, J. Exp. Med., 2005, 202, 1261-1269. 
12 H. Takayanagi, S. Kim, T. Koga, H. Nishina, M. Isshiki, H. Yoshida, et al., Induction and activation of the transcription factor NFATc1 (NFAT2) integrate RANKL signaling in terminal differentiation of osteoclasts, Dev. Cell, 2002, 3, 889-901.

13 H. Feng, T. Cheng, J. H. Steer, D. A. Joyce, N. J. Pavlos, C. Leong, et al., Myocyte enhancer factor 2 and microphthalmia-associated transcription factor cooperate with NFATc1 to transactivate the V-ATPase $\mathrm{d} 2$ promoter during RANKL-induced osteoclastogenesis, J. Biol. Chem., 2009, 284, 14667-14676.

14 K. Scherlach, D. Boettger, N. Remme and C. Hertweck, The chemistry and biology of cytochalasans, Nat. Prod. Rep., 2010, 27, 869-886.

15 R. Liu, Z. Lin, T. Zhu, Y. Fang, Q. Gu and W. Zhu, Novel openchain cytochalsins from the marine-derived fungus Spicaria elegans, J. Nat. Prod., 2008, 71, 1127-1132.

16 M. Trendowski, J. M. Mitchell, C. M. Corsette, C. Acquafondata and T. P. Fondy, Chemotherapy with cytochalasin congeners in vitro and in vivo against murine models, Invest. New Drugs, 2015, 33, 290-299.

17 R. M. Samsonraj, A. Dudakovic, B. Manzar, B. Sen, A. B. Dietz, S. M. Cool, et al., Osteogenic Stimulation of Human Adipose-Derived Mesenchymal Stem Cells Using a Fungal Metabolite That Suppresses the Polycomb Group Protein EZH2, Stem Cells Transl. Med., 2018, 7, 197-209.

18 S. Xu, H. M. Ge, Y. C. Song, Y. Shen, H. Ding and R. X. Tan, Cytotoxic cytochalasin metabolites of endophytic Endothia gyrosa, Chem. Biodiversity, 2009, 6, 739-745.

19 H. W. Zhang, J. Zhang, S. Hu, Z. J. Zhang, C. J. Zhu, S. W. Ng, et al., Ardeemins and cytochalasins from Aspergillus terreus residing in Artemisia annua, Planta Med., 2010, 76, 16161621.

20 J. Xu, J. W. Tan, L. Huang, X. H. Gao, R. Laird, D. Liu, et al., Cloning, sequencing, and functional characterization of the rat homologue of receptor activator of NF-kappaB ligand, $J$. Bone Miner. Res., 2000, 15, 2178-2186.

21 K. Chen, Y. Yuan, Z. Wang, D. Song, J. Zhao, Z. Cao, et al., Helvolic acid attenuates osteoclast formation and function via suppressing RANKL-induced NFATc1 activation, J. Cell. Physiol., 2019, 234, 6477-6488.

22 L. Zhou, Q. Liu, M. Yang, T. Wang, J. Yao, J. Cheng, et al., Dihydroartemisinin, an Anti-Malaria Drug, Suppresses Estrogen Deficiency-Induced Osteoporosis, Osteoclast Formation, and RANKL-Induced Signaling Pathways, $J$. Bone Miner. Res., 2016, 31, 964-974.
23 M. Inoue, F. P. Ross, J. M. Erdmann, Y. Abu-Amer, S. Wei and S. L. Teitelbaum, Tumor necrosis factor alpha regulates alpha(v)beta5 integrin expression by osteoclast precursors in vitro and in vivo, Endocrinology, 2000, 141, 284-290.

24 Q. Zhao, X. Wang, Y. Liu, A. He and R. Jia, NFATc1: functions in osteoclasts, Int. J. Biochem. Cell Biol., 2010, 42, 576-579.

25 K. Matsuo, D. L. Galson, C. Zhao, L. Peng, C. Laplace, K. Z. Wang, et al., Nuclear factor of activated T-cells (NFAT) rescues osteoclastogenesis in precursors lacking cFos, J. Biol. Chem., 2004, 279, 26475-26480.

26 S. L. Teitelbaum, Bone resorption by osteoclasts, Science, 2000, 289, 1504-1508.

27 J. F. Charles and A. O. Aliprantis, Osteoclasts: more than 'bone eaters', Trends Mol. Med., 2014, 20, 449-459.

28 E. Verron and J. M. Bouler, Is bisphosphonate therapy compromised by the emergence of adverse bone disorders?, Drug Discovery Today, 2014, 19, 312-319.

29 K. A. Cappuzzo and J. C. Delafuente, Teriparatide for severe osteoporosis, Ann. Pharmacother., 2004, 38, 294-302.

30 T. Wada, T. Nakashima, N. Hiroshi and J. M. Penninger, RANKL-RANK signaling in osteoclastogenesis and bone disease, Trends Mol. Med., 2006, 12, 17-25.

31 H. Hsu, D. L. Lacey, C. R. Dunstan, I. Solovyev, A. Colombero, E. Timms, et al., Tumor necrosis factor receptor family member RANK mediates osteoclast differentiation and activation induced by osteoprotegerin ligand, Proc. Natl. Acad. Sci. U. S. A., 1999, 96, 3540-3545.

32 M. Matsumoto, M. Kogawa, S. Wada, H. Takayanagi, M. Tsujimoto, S. Katayama, et al., Essential role of p38 mitogen-activated protein kinase in cathepsin $\mathrm{K}$ gene expression during osteoclastogenesis through association of NFATc1 and PU.1, J. Biol. Chem., 2004, 279, 45969-45979.

33 H. Takatsuna, M. Asagiri, T. Kubota, K. Oka, T. Osada, C. Sugiyama, et al., Inhibition of RANKL-induced osteoclastogenesis by (-)-DHMEQ, a novel NF-kappaB inhibitor, through downregulation of NFATc1, J. Bone Miner. Res., 2005, 20, 653-662.

34 V. Iotsova, J. Caamano, J. Loy, Y. Yang, A. Lewin and R. Bravo, Osteopetrosis in mice lacking NF-kappaB1 and NF-kappaB2, Nat. Med., 1997, 3, 1285-1289.

35 T. Negishi-Koga and H. Takayanagi, $\mathrm{Ca}^{2+}$-NFATc1 signaling is an essential axis of osteoclast differentiation, Immunol. Rev., 2009, 231, 241-256.

36 E. F. Wagner and R. Eferl, Fos/AP-1 proteins in bone and the immune system, Immunol. Rev., 2005, 208, 126-140. 